\title{
Characterization and Calibration of PILATUS Detectors
}

\author{
P. Kraft, A. Bergamaschi, Ch. Brönnimann, R. Dinapoli, E. F. Eikenberry, H. Graafsma, B. Henrich, I. Johnson, \\ M. Kobas, A. Mozzanica, C. M. Schlepütz, and B. Schmitt
}

\begin{abstract}
Pilatus is a silicon hybrid pixel detector system for detecting X-rays in single photon counting mode. The PILATUS II chip, fabricated in a radiation tolerant design with a standard $0.25 \mu \mathrm{m}$ CMOS process, was used to construct multichip modules with a size of $84 \times 34 \mathrm{~mm}^{2}$ comprising 94 '965 pixels. All calibrations and characterizations were carried out with monochromatic $\mathrm{X}$-rays from a synchrotron source. In order to set any required threshold above the noise level between $2.14 \mathrm{keV}$ and $22 \mathrm{keV}$ the detector was calibrated with X-rays. An algorithm to adjust thresholds pixel-by-pixel and create trim files based on X-ray flat-field images was developed. The threshold dispersion was reduced from $343 \mathrm{eV}$ to $36 \mathrm{eV}$ by the means of trim files. An electronic noise of $447 \mathrm{eV}$ has been measured. The PILATUS modules are suitable for various $\mathrm{X}$-ray applications such as diffraction and imaging techniques.
\end{abstract}

Index Terms-Hybrid pixel detector, single photon counting, threshold calibration, threshold dispersion, threshold trimming, noise.

\section{INTRODUCTION}

$\mathbf{P}$ ILATUS is a silicon hybrid pixel detector system for $\mathrm{X}$-rays operated at room temperature, using bump and flip-chip technology ${ }^{1}$, which has been developed at the Paul Scherrer Institut (PSI) [1], [2]. It was purpose-built for macromolecular crystallography at the Swiss Light Source (SLS) [3].

A detector for macromolecular crystallography needs to measure several thousands of reflections in a single diffraction image which span over five orders of magnitude difference in integrated intensity between the weakest and strongest. This demands a detector with a high dynamic range, high count rate capability, high detection efficiency and a very good point spread function to achieve the best possible signal to noise ratio. Furthermore a fast readout is important to speed up experiments in order to avoid radiation damage in the sample and increase the throughput at the beamline. Single photon counting hybrid pixel detectors are well suited to meet these requirements. The

Manuscript received June 30, 2008; revised October 21, 2008. Current version published June 17, 2009.

P. Kraft, A. Bergamaschi, R. Dinapoli, B. Henrich, I. Johnson, A. Mozzanica, C. M. Schlepütz, and B. Schmitt are with the Paul Scherrer Institut, 5232 Villigen PSI, Switzerland (e-mail: philipp.kraft@psi.ch).

C. Brönnimann, E. F. Eikenberry, and M. Kobas are with the DECTRIS, Ltd., 5400 Baden, Switzerland.

H. Graafsma is with the HASYLAB, DESY, 22603 Hamburg, Germany.

Color versions of one or more of the figures in this paper are available online at http://ieeexplore.iee.org.

Digital Object Identifier 10.1109/TNS.2008.2009448

${ }^{1}$ Each readout channel is electrically connected to its corresponding detecting element through a microscopic indium ball. single photon counting approach disposes of dark currents and readout noise effects which are typical for integrating detectors, such as CCDs (charge coupled devices). The information is stored digitally in the pixel and can be readout quickly.

The presented PILATUS II readout chip (ROC) is a significant improvement of its predecessor the PILATUS I chip [4].

The charge pulse generated in the sensor by an incident photon is compared to a threshold before the counter is triggered. This single level threshold features an energy selection. The pixel threshold is ideally kept at 50\% of the incident X-ray energy to avoid double counting at pixel boarders and to reach the highest possible detection efficiency. Thus a precise energy calibration of the threshold in combination with a small pixel to pixel threshold dispersion is necessary.

Besides macromolecular crystallography PILATUS meets the typical requirements of several diffraction and imaging experiments as powder diffraction [5], surface diffraction [6], phase contrast imaging [7] and dark-field imaging [8]. Detectors of any size with a multiple of $487 \times 195$ active pixels can be realized with arrays of PILATUS modules.

We describe the architecture and the functional behavior of the PILATUS modules in the second section. Characterizations and calibrations with $\mathrm{X}$-rays regarding threshold dispersion, electronic noise and threshold trimming are presented in the third section.

\section{SYSTEM DESCRIPTION}

\section{A. The Readout Chip}

The PILATUS II readout chip was improved in terms of dynamic range, count rate capability, pixel size, readout speed and stability. It is designed in a standard $0.25 \mu \mathrm{m}$ CMOS process using radiation tolerant layout techniques [9], [10] and it has been available since late 2004. The principal properties of the ROC are listed in Table I and a scheme of the chip and the pixel cell architecture respectively is shown in Fig. 1.

The ROC features single photon counting at room temperature [11]. This means that the charge pulse generated in the sensor by conversion of an incident photon is collected and amplified by a two stage amplifier consisting of a charge sensitive preamplifier (CSA) and an AC coupled shaper to reduce electronic noise. The shaper is then followed by a single level comparator (Comp) producing a digital signal if the pulse amplitude exceeds the given threshold. The comparator threshold of each pixel is adjusted with a global threshold voltage (VCMP) and can be individually trimmed with a 6 bit digital-to-analog converter (6 bit DAC). A digital pulse from the comparator incre- 


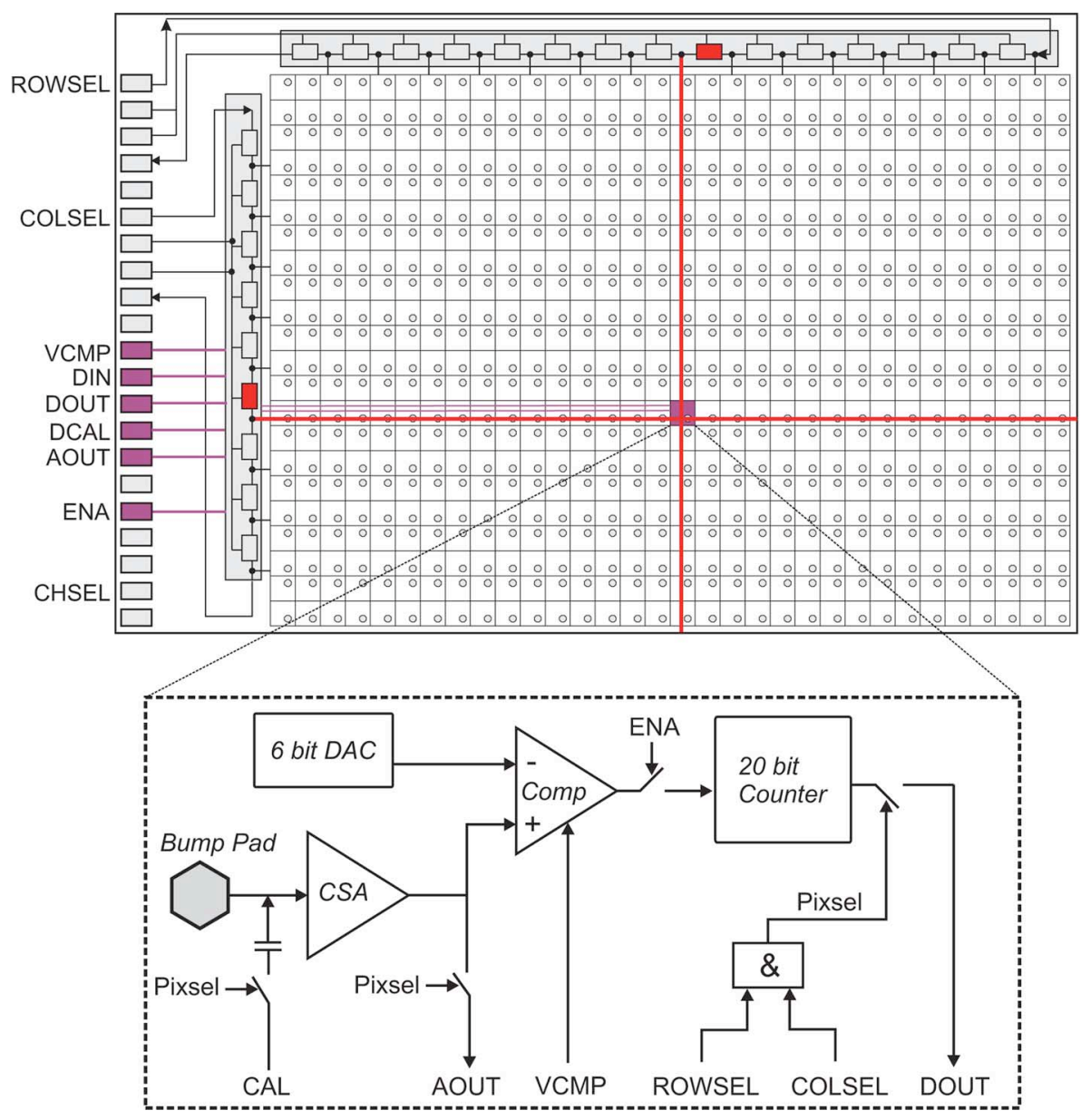

Fig. 1. Architecture of the PILATUS II readout chip (top) and pixel cell (bottom).

TABLE I

PRINCIPAL PROPERTIES OF THE PILATUS II READOUT CHIP

\begin{tabular}{ll}
\hline Design & $0.25 \mu \mathrm{m}$ (radiation tolerant layout) \\
Pixel format & $60 \times 97=5820$ \\
Pixel size & $172 \times 172 \mu \mathrm{m}^{2}$ \\
Chip size & $17.54 \times 10.45 \mathrm{~mm}^{2}$ \\
Dynamic range & $1^{\prime} 0488^{\prime} 574(20 \mathrm{bit}$ counter/pixel) \\
Clock frequency & $66 \frac{2}{3} \mathrm{MHz}$ \\
Readout time & $2.85 \mathrm{~ms}$ \\
Threshold adjustment & $6 \mathrm{bit} \mathrm{DAC} /$ pixel \\
\hline
\end{tabular}

ments the 20 bit counter, leading to completely digital storage of the number of detected X-rays in each pixel.

The shaper analog output (AOUT) of each pixel can be multiplexed onto the analog out bus of the column and from there to the analog output of the chip. In test mode a digital calibration signal (DCAL) can be sent to each pixel where a transistor is used to charge a capacitor connected to the input of the CSA. Thus a charge pulse (CAL) is injected into the analog front end simulating a converted photon in the sensor.

Every pixel is individually addressable with the Pixsel signal given by an AND gate from the signals ROWSEL and COLSEL. The ROWSEL and COLSEL signals are controlled by the row selection shift register and the column selection shift register respectively, located in the chip periphery. A pixel needs to be addressed in order to read from and write to its counter (DOUT/ DIN), to program the 6 bit DAC, to send calibrate signals (CAL) to its CSA input and to monitor its analog output (AOUT).

Two main modes of operation are defined: In count mode, no pixel is selected and all pixels are individually counting X-rays while the external enable signal (ENA) is logic high, acting as an electronic shutter. During readout mode each pixel is sequentially selected and the stored data in its counter transferred to the digital output (DOUT). With a clock frequency of $66(2 / 3) \mathrm{MHz}$ the readout time for the complete ROC is $2.85 \mathrm{~ms}$. An entire ROC is enabled using a chip select signal (CHSEL) such that chips on a multichip module can be addressed individually. Details regarding the testing and characterization of the PILATUS II readout chip may be found in [12].

\section{B. The Sensor}

The silicon sensors (Fig. 2) used for the PILATUS modules were designed at PSI and fabricated by HAMAMATSU [13]. Each pixel consists of a pn-junction realized by a highly doped p-electrode implanted into a high-resistivity n-bulk as shown in Fig. 3. The size of the p-implant is $112 \mu \mathrm{m} \times 112 \mu \mathrm{m}$ with a pixel pitch of $172 \mu \mathrm{m} \times 172 \mu \mathrm{m}$. The implants are contacted by aluminum pads through vias in the oxide. The whole sensor is passivated with $\sim 1 \mu$ m-thick $\mathrm{SiO}_{2}$ opened only at the positions 


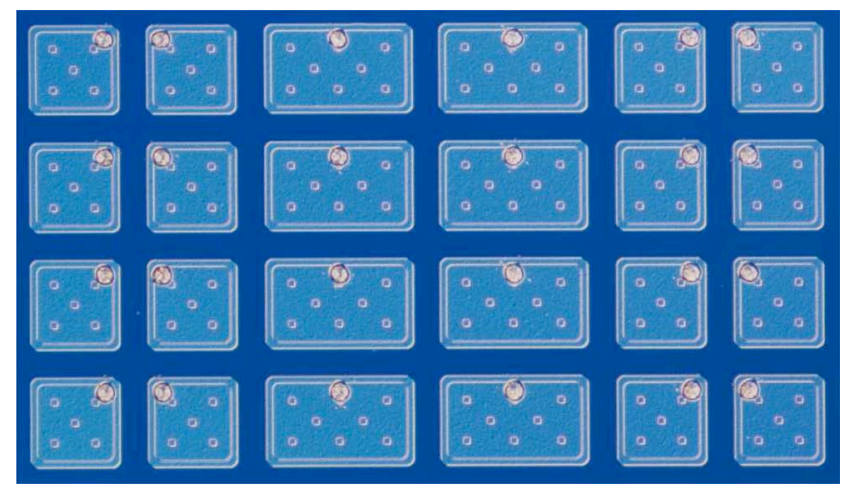

Fig. 2. Light microscope image of the sensor flip side with normal pixels (square) and edge pixels (rectangular). The edge pixels span the gaps between neighboring chips avoiding dead area.

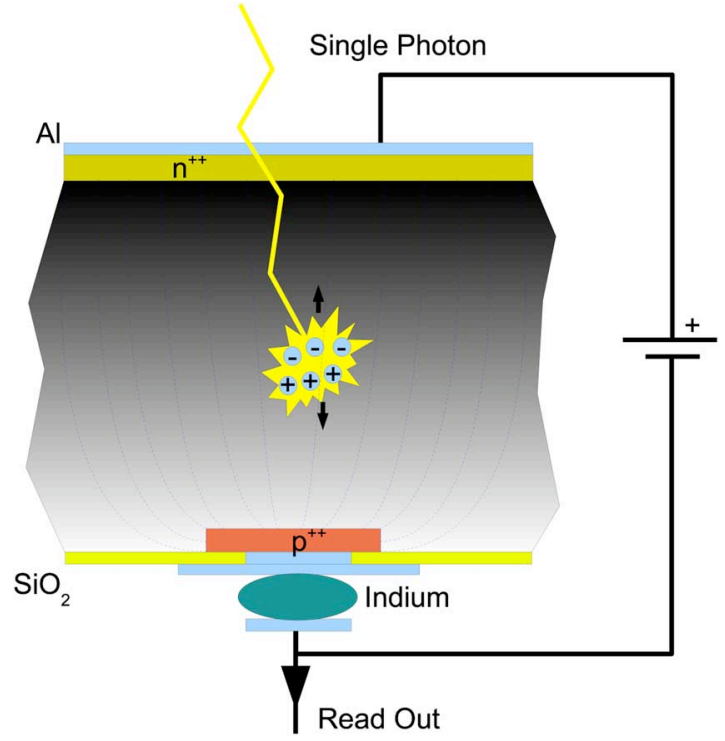

Fig. 3. Scheme of sensor pixel architecture with converting photon in electric field.

of the bump pads. The opposite surface is unstructured and consists of an aluminum layer contacting a highly doped n-implant. The Al layer also protects the sensor from visible light. The PILATUS sensors with a thickness of $320 \mu \mathrm{m}$ are fully depleted at about $80 \mathrm{~V}$ and normally biased with $120 \mathrm{~V}$.

Sensor and readout chip are optimized for hole collection. The average number of generated electron-hole pairs $N_{e h}$ in the Silicon is proportional to the X-ray energy $E_{\gamma}, N_{e h} \approx$ $E_{\gamma} / 3.63 \mathrm{eV}$ [14].

\section{The Module}

A PILATUS detector module consists of a single, fully depleted monolithic silicon sensor bump-bonded to an array of $8 \times 2$ ROCs. A model of the hybrid architecture is shown in Fig. 4. Each sensor pixel is electrically connected to its corresponding ROC pixel with an Indium bump ball of 15 to $25 \mu \mathrm{m}$ diameter. Wirebonds are used to connect the pads on the side of the ROC to further readout electronics.

Two rows or columns of larger pixels with a size of $3 / 2$ of a normal pixel $(258 \mu \mathrm{m} \times 172 \mu \mathrm{m})$ span the gaps between

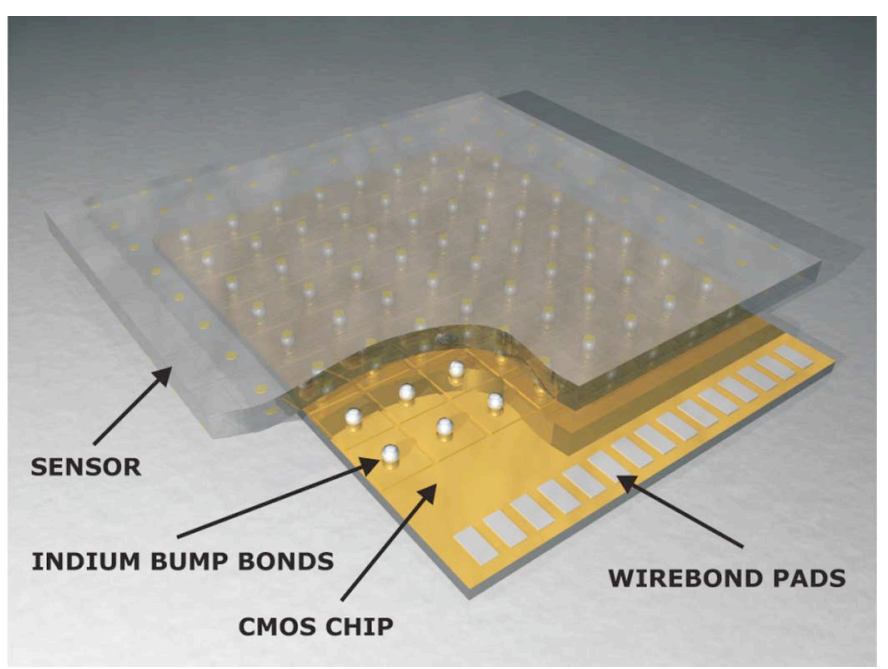

Fig. 4. Model of hybrid architecture.

ROCs (Fig. 2), thus there is no dead area between chips. The registered counts of these larger pixels are distributed by software into three normal sized pixels by introducing a virtual pixel in between. The same principle is applied in the area where the corners of four ROCs meet. In this case the gap is covered by four large pixels with the size of $9 / 4$ of a normal pixel $(258 \mu \mathrm{m} \times 258 \mu \mathrm{m})$. Introducing five virtual pixels and distributing the counts accordingly delivers nine normal sized pixels. Thus a continuous array of $487 \times 195=94^{\prime} 965$ pixels per module of identical pitch $(172 \mu \mathrm{m} \times 172 \mu \mathrm{m})$ is achieved. The module covers an active area of $83.78 \mathrm{~mm} \times 33.56 \mathrm{~mm}$. The active area of the sensor is surrounded by a guard ring of $100 \mu \mathrm{m}$ width contacted to ground via bump bonds on the ROC. The guard ring is necessary to stabilize the sensor by drawing off the edge leakage current.

The bare module (sensor and chips) is attached to a $200-\mu \mathrm{m}$ -thick silicon baseplate for mechanical and thermal stabilization. This assembly is cemented and wirebonded onto a high density interconnect (HDI), which sits on a mounting bracket yielding a total module area of $84.90 \mathrm{~mm} \times 36.34 \mathrm{~mm}$ (Fig. 5). The module control board (MCB) is plugged into the back side of the HDI through holes in the bracket. The MCB contains the regulators for the supply voltages, the DACs for the global biasing of the ROCs and a connector for further readout electronics. An $\mathrm{I}^{2} \mathrm{C}$ serial interface is used to control the DACs [15]. The comparator threshold can be programmed for each chip individually, while all the other bias voltages are common for the entire module. The addressing of the chips, which is important for readout, threshold trimming and debugging, is also accomplished by the $\mathrm{I}^{2} \mathrm{C}$ interface. During normal operation the necessary steering signals are distributed by the MCB to all the chips in parallel. Moreover, the MCB supports parallel readout of the 16 chips.

\section{Detector Systems}

One module together with a detector control board (DCB), a data acquisition computer and a power supply forms a standalone detector system, the PILATUS $100 \mathrm{~K}$. The DCB services 


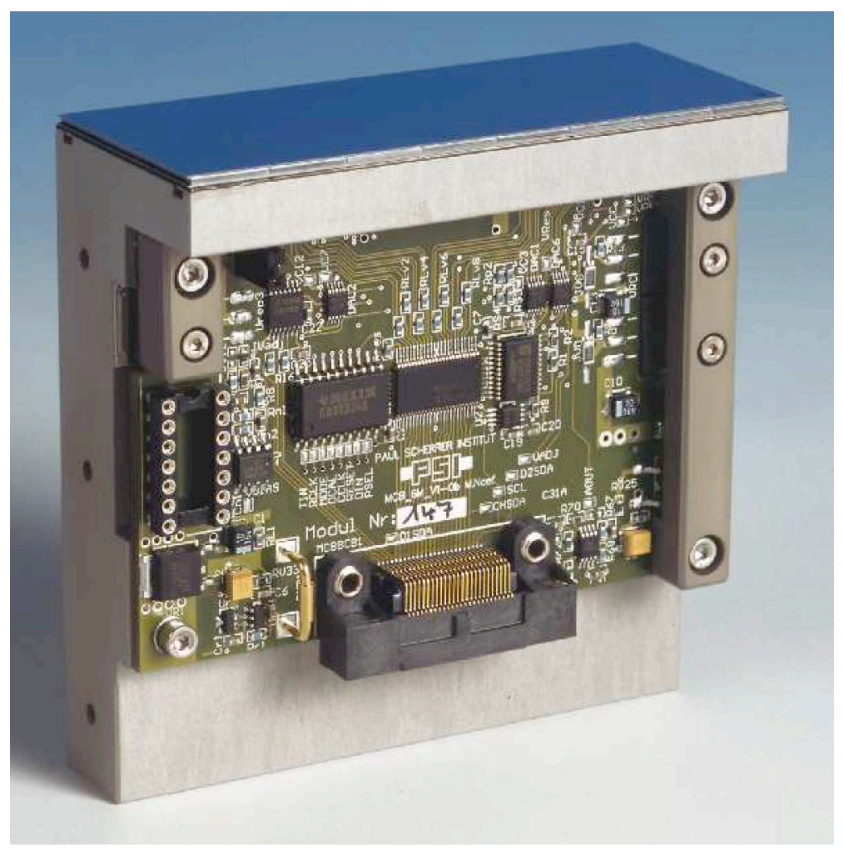

Fig. 5. PILATUS detector module on mounting bracket with module control board.

the MCB, serializes and transfers the data to a PCI card on the computer side. With the PILATUS $100 \mathrm{~K}$ detector a maximum frame rate of $200 \mathrm{~Hz}$ is achieved. A $100 \mathrm{~K}$ system was used for the characterizations and calibrations presented in this paper.

A multi-module setup of any array size can be realized by mounting modules on a high-precision mechanical frame. Several modules are grouped to form a bank and connected to a bank control board (BCB). The $\mathrm{BCB}$ features parallel readout of the modules and temporarily stores the data until they are fetched by the DCB [16]. Two different configurations were fabricated and commissioned at PSI for the SLS. The PILATUS $6 \mathrm{M}$ is an array of $5 \times 12$ modules comprising 6'224'001 pixels on an active area of $431 \mathrm{~mm} \times 448 \mathrm{~mm}$ achieving $12.5 \mathrm{~Hz}$ maximum frame rate. It is used at the macromolecular crystallography beamline X06SA at the SLS. The PILATUS $2 \mathrm{M}$ is an array of $3 \times 8$ modules comprising 2'476' 525 pixels on an active area of $254 \mathrm{~mm}$ $\times 289 \mathrm{~mm}$ achieving $30 \mathrm{~Hz}$ maximum frame rate. This detector is in use at the coherent small-angle X-ray scattering beamline $\mathrm{X} 12 \mathrm{SA}$.

\section{CHARACTERIZATION AND CALIBRATION}

All presented calibrations and characterizations were carried out at the SLS using monochromatic X-rays since better results were achieved with X-rays than with the internal calibration signal (CAL) (Fig. 1) of the readout chip. Either the direct synchrotron beam in combination with absorbing filters or an elastic scatterer for homogeneous detector illumination were used. The monochromaticity of the source was inspected with an AMPTEK X-123 spectrometer [17].

\section{A. Charge Sensitive Amplifier}

The analog signal (AOUT) (Fig. 1) of each pixel can be investigated with an oscilloscope. This is very useful to optimize the
CSA performance for specific requirements although the pristine AOUT signal is slightly distorted by the analog output circuit of the chip. A certain CSA setting means always a trade-off between different detector characteristics in terms of threshold range, energy resolution, threshold dispersion and rate capability.

Gain and shaping time of the CSA are adjusted with a global bias voltage $\left(V_{r f}\right)$, whereas amplitude and width of the output pulses are correlated such that more gain produces larger and wider pulses. Any $V_{r f}$-value between $-0.6 \mathrm{~V}$ and $-0.03 \mathrm{~V}$ can be chosen to fulfill certain experimental requirements. Each setting requires an individual energy threshold calibration, threshold trimming and rate correction.

The CSA settings may be adjusted to suit the needs of the experiments. The low gain settings $\left(V_{r f}=-0.3 \mathrm{~V}\right)$ are devoted to high rate experiments with up to $8 \cdot 10^{6}$ incident photons per second per pixel (ph/s/pixel). For many applications the medium gain settings $\left(V_{r f}=-0.2 \mathrm{~V}\right)$ are suitable since the mentioned detector characteristics are well balanced. To access the low energy range the high gain settings $\left(V_{r f}=-0.15 \mathrm{~V}\right)$ are employed but the incident rate must stay below $0.9 \cdot 10^{6} \mathrm{ph} / \mathrm{s} /$ pixel. The corresponding waveforms of the AOUT signal for these CSA settings are shown in Fig. 6.

\section{B. Comparator}

1) Threshold Scan: The pulse height from the shaper output signal is compared with the pixel threshold of the comparator. The external threshold voltage $\left(V_{c m p}\right)$ is global for a whole chip and usually kept common for an entire module (Fig. 1).

In order to characterize and calibrate $V_{c m p}$ so-called threshold scans are carried out. For that purpose the module is homogeneously illuminated with X-rays of a certain energy. Images of equal exposure time are taken while the global threshold ${ }^{2}$ is decreased with respect to energy for each frame. The threshold scans of a pixel for two different X-ray energies are shown in Fig. 7.

The threshold scan of a perfect detector would follow a step function with the transition centered at the incident X-ray energy, in case of a perfectly monochromatic source. Mainly because of the electronic noise of the PILATUS pixel the transition in the scans is smeared out around the incident X-ray energy. Furthermore the count rate keeps constantly growing below the $\mathrm{X}$-ray energy due to charge sharing in the sensor ${ }^{3}$.

The s-curve method presented in [10] is used to analyze the threshold scans. The s-curve is extended with a linear term multiplied by the erf() function in order to take into account the charge sharing contribution:

$$
n\left(V_{c m p}\right)=\frac{1}{2} \operatorname{erf}\left(\frac{V_{c m p}-a_{1}}{\sqrt{2} a_{2}}\right)\left(a_{3}+a_{4}\left(V_{c m p}-a_{1}\right)\right) .
$$

Function (1) has a well defined inflection point $a_{1}$ which is the threshold of the pixel for the given X-ray energy. The parameter $a_{2}$ is related to the electronic noise, charge sharing and energy spectrum of the incident $\mathrm{X}$-rays. The amplitude of the error

\footnotetext{
${ }^{2} \mathrm{An}$ increase in $V_{c m p}$ means a decrease in threshold.

${ }^{3}$ Sharing of signal charge between neighboring pixels [18].
} 


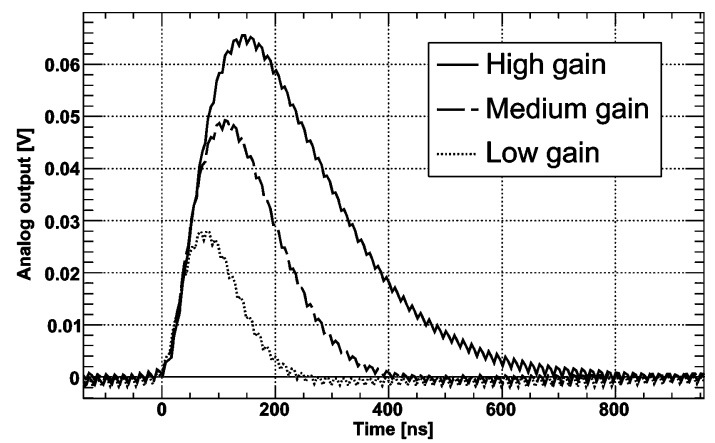

Fig. 6. Analog output of a pixel for $6.4 \mathrm{keV} \mathrm{X-rays} \mathrm{and} \mathrm{three} \mathrm{different} \mathrm{CSA}$ settings.

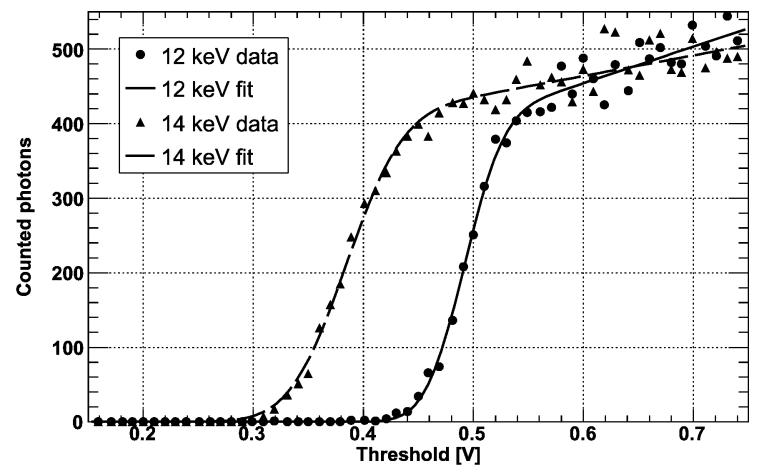

Fig. 7. Threshold scans of a pixel for two different $\mathrm{X}$-ray energies at low gain CSA settings.

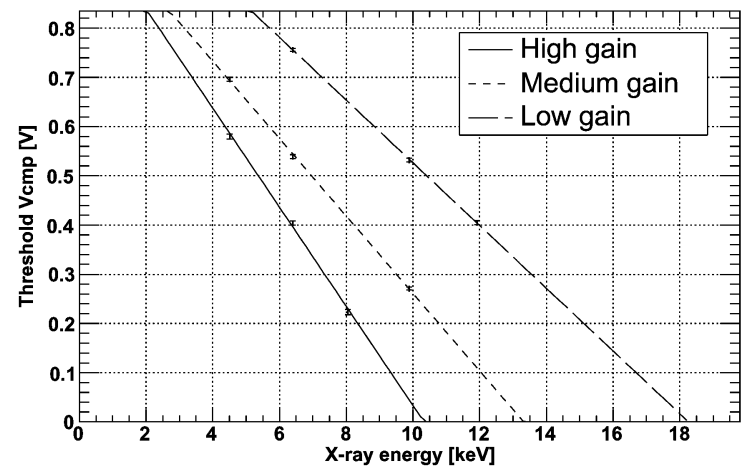

Fig. 8. Global threshold calibration of a pixel for three different CSA gain settings.

function $a_{3}$ depends on the flux of the source and the exposure time. The slope $a_{4}$ in the linear term models the charge sharing.

2) Global Threshold Calibration: The energy calibration of $V_{c m p}$ for a pixel can be obtained by repeating threshold scans at different X-ray energies. For each scan the threshold $a_{1}$ is obtained by fitting (1) to the data. The pixel threshold values of $V_{c m p}$ are plotted against the corresponding X-ray energy $E_{\gamma}$ and fitted with a straight line, since the comparator was designed to be linear:

$$
V_{c m p}\left(E_{\gamma}\right)=s E_{\gamma}+o,
$$

where $s$ designates the slope and $o$ the offset of the straight line. The $V_{c m p}$ values and resulting linear fits of a pixel are shown for three different CSA gain settings in Fig. 8.

The values of slope and offset averaged over all pixels of a module yield the global threshold calibration for the used CSA
TABLE II

Global Threshold Calibration of A Module For THREE DifFERENT CSA GAIN SETTINGS

\begin{tabular}{lcc} 
CSA setting & Slope $[\mathrm{mv} / \mathrm{keV}]$ & Offset $[\mathrm{mV}]$ \\
\hline High Gain & $-116.49 \pm 0.06$ & $1^{\prime} 107.6 \pm 0.07$ \\
Medium Gain & $-84.59 \pm 0.05$ & $1 ' 051.7 \pm 0.06$ \\
Low Gain & $-64.69 \pm 0.05$ & $1 ' 106.0 \pm 0.06$ \\
\hline
\end{tabular}

TABLE III

THRESHOLD DISPERSION OF A (UN)TRIMMED MODULE FOR DIFFERENT X-RAY ENERGIES at Low Gain CSA SetTings $\left(V_{r f}=-0.3 \mathrm{~V}\right) \mathrm{IN} \mathrm{eV}$

\begin{tabular}{rcc} 
Energy $[\mathrm{keV}]$ & Untrimmed & Trimmed \\
\hline 8 & $429 \pm 13$ & - \\
10 & $493 \pm 15$ & $58 \pm 14$ \\
12 & $561 \pm 15$ & $58 \pm 15$ \\
14 & $634 \pm 16$ & $65 \pm 16$ \\
16 & - & $74 \pm 17$ \\
\hline
\end{tabular}

gain settings. The global threshold calibration of a module is shown in Table II for three different CSA gain settings. Above $V_{c m p}=0.8 \mathrm{~V}$ the threshold reaches the electronic noise level of the pixel. This value changes slightly with different CSA gain settings. A calibration for ultra-high gain settings $\left(V_{r f}=\right.$ $-0.03 \mathrm{~V}$ ) was obtained in order to determine the least possible threshold of a module. It was found that the maximum $V_{c m p}$ at which all the pixels of the module were still counting above the electronic noise corresponds to a minimum threshold of $2.14 \mathrm{keV}$.

Only the slope is of use for the fully calibrated module since the offset is automatically given by the trim files as described in Section III-C-I.

3) Intrinsic Threshold Dispersion: The global threshold calibration can be used to convert the $V_{c m p}$ pixel threshold value which was obtained from a threshold scan into energy. The histogram of these pixel threshold energy values is the threshold distribution of the module. And the root mean square (r. m. s.) of this distribution is the threshold dispersion of the module for the used X-ray energy. The threshold dispersion in the module originates from fabrication inhomogeneities and voltage drops along the power distribution lines in the read out chip. It can be considered as a part stemming from the gain dispersion of the CSA which depends on the pulse height and thus on the energy of the incident photons and another constant part which is energy independent. We found that the intrinsic threshold dispersion of a module for $8 \mathrm{keV} \mathrm{X-rays} \mathrm{at} \mathrm{high} \mathrm{gain} \mathrm{CSA} \mathrm{settings}$ was $343 \pm 10 \mathrm{eV}$ and for $10 \mathrm{keV} \mathrm{X}$-rays at medium gain CSA settings $490 \pm 10 \mathrm{eV}$. The results for different $\mathrm{X}$-ray energies and low gain CSA gain settings are shown in Table III.

4) Electronic Noise: As stated in Section III-B-I the parameter $a_{2}$ in (1) is related to the electronic noise, the charge sharing of the pixel and the energy spectrum of the incident $\mathrm{X}$-rays. Therefore threshold scan data using monochromatic $\mathrm{X}$-rays which is free of charge sharing is necessary in order to measure the electronic noise with $a_{2}$ from the fit.

According to [18] the charge sharing occurs at the border between neighboring pixels. Thus two laser drilled Mo grids with squares corresponding to the pitch of the pixel were fabricated. The grids were mounted in front of each other on piezo 
tables as close to the detector sensor as possible. The grids were aligned such that the resulting appertures were $80 \mu \mathrm{m} \times 80 \mu \mathrm{m}$ providing a pencil X-ray beam on every pixel center. Using an elastically scattered synchrotron beam in combination with this setup, we were able to carry out threshold scans without any charge sharing in order to determine the noise of the pixel with the following method.

The derivative of the error function in (1) yields a Gaussian curve

$$
\frac{d}{d V_{c m p}} \frac{a_{3}}{2} \operatorname{erf}\left(\frac{V_{c m p}-a_{1}}{\sqrt{2} a_{2}}\right)=\frac{a_{3}}{\sqrt{2 \pi} a_{2}} e^{-\left(V_{c m p}-a_{1}\right)^{2} / 2 a_{2}^{2}}
$$

with $a_{2}$ being its standard deviation. This Gaussian peak corresponds to the spectrum of the X-ray source seen by a pixel including its electronic noise. With the beamline's energy resolution of $\Delta E / E<0.02 \%$ the contribution from the $\mathrm{X}$-ray source is negligible. Therefore the width of the peak is only given by the noise and $a_{2}$ is a measure for the r. m. s. electronic noise of the pixel including sensor, analog and digital part. The measured average electronic noise (r. m. s.) of the pixel in case of low gain CSA settings and $8 \mathrm{keV} \mathrm{X}$-rays is $447 \pm 3 \mathrm{eV}=123 \pm 1 \mathrm{e}^{-}$.

\section{Trim DAC}

The global threshold can be trimmed with the 6 bit DAC in each pixel to correct the threshold dispersion described in Section III-B-III. The DAC is programmable with selected bits of the 20-bit shift register in the counter. The output of the DAC is added such that the global threshold given by $V_{c m p}$ is elevated. The strength of the trim-bits with respect to the global threshold is set with the bias $V_{t r m}$ which is global for the entire module.

1) Threshold Trimming Procedure: To determine the individual trim-bit combination for each pixel a threshold trimming procedure was developed. During the whole procedure the module needs to be irradiated with monochromatic X-rays corresponding to the target threshold energy. The global threshold $\left(V_{c m p}\right)$ needs to be set below the X-ray energy such that all pixels are fully counting with all the trim-bits turned off. Then all trim-bits are enabled and images are taken with increasing trim-bit strength $\left(V_{t r m}\right)$ until all the pixels are counting very little of the incident photons. After these steps the target threshold of each pixel lies within the range of the trim-bits. During a trim scan the 64 possible trim-bit combinations are sequentially programmed in all the pixels and an image of equal exposure time is taken for each combination. Subsequently the trim scan data of each pixel following a s-curve is fitted and the trim-bit value closest to the inflection point is stored in a trim file. The thereby obtained trim-bit value corresponds to the offset of the individual pixel threshold from the target threshold. The quality of the trim files and the correction of the threshold dispersion is strongly influenced by counting statistics and the monochromaticity of the X-rays.

The offset on the global threshold $\left(V_{c m p}\right)$ given by the trim DACs changes the average offset of the global threshold calibration but not its average slope. Thus the average slope can be used to calculate the required shift in $V_{c m p}$ in order to move

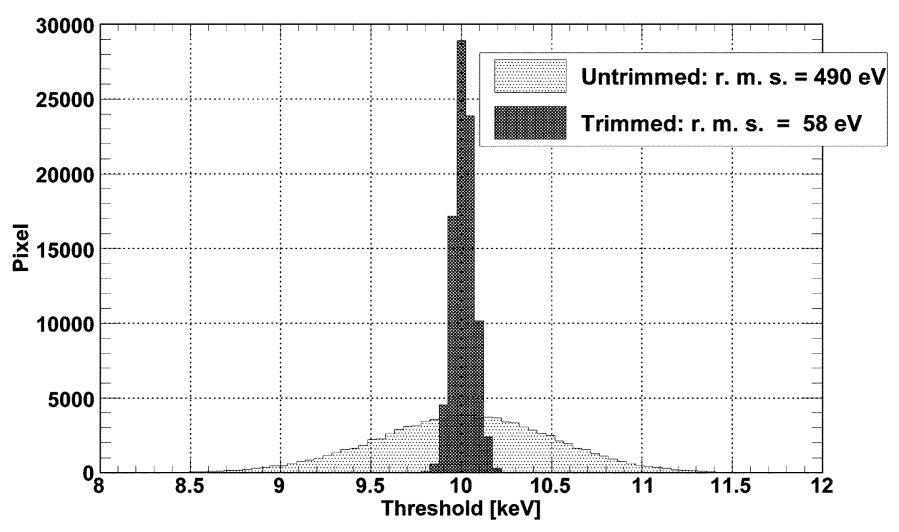

Fig. 9. Threshold distribution of a trimmed module for $10 \mathrm{keV}$ X-rays with low gain CSA settings in comparison with the untrimmed distribution.

the trimmed target threshold energy. Since the threshold dispersion is energy dependent (Section III-B-III) a trim file applies only for the target threshold energy it was actually produced at, i.e., changing $V_{c m p}$ will enlarge the threshold dispersion. In the case of medium gain CSA settings an increase of the threshold dispersion by $33 \pm 1.4 \mathrm{eV}$ was measured for a difference of $1 \mathrm{keV}$ between global threshold and trimmed target threshold. Since the trimming procedure is time consuming we produce trim files for target threshold energies at intervals of $2 \mathrm{keV}$ and adjust $V_{c m p}$ to interpolate in between. This method complies with most of the experimental requirements.

2) Corrected Threshold Dispersion: The quality of trim files is investigated by the means of threshold scans taken with a trimmed module using X-rays of the trim target threshold energy. For the conversion from $V_{c m p}$ to energy the previously determined global threshold calibration is employed. The average of the trimmed threshold distribution determines the average offset given by the trim DACs. The threshold distributions from two threshold scans at $10 \mathrm{keV} \mathrm{X}$-ray energy are shown for low gain CSA settings in Fig. 9. The grey histogram represents the threshold distribution of the untrimmed module and the black distribution is the result of a trimmed threshold scan. The threshold dispersion was in this case improved by more than a factor of 8 .

The above described method to inspect the success of the threshold trimming procedure was applied to a module for different CSA gain settings and at different target threshold energies. For $8 \mathrm{keV} \mathrm{X}$-rays we measured $36 \pm 10 \mathrm{eV}$ for high gain and $48 \pm 12 \mathrm{eV}$ for medium gain CSA settings. The resulting threshold dispersions for low gain CSA settings are listed in Table III. Due to the limited range of the comparator the dispersions for $16 \mathrm{keV}$, untrimmed and $8 \mathrm{keV}$, trimmed are missing.

In order to minimize double counting from charge sharing and maximize the detection efficiency a threshold at exactly half of the X-ray energy is favorable for most experiments. A higher threshold can be used to discriminate against fluorescence background at the expense of efficiency.

\section{Discussion AND CONCLUSION}

Single photon counting requires accurate energy calibration and threshold trimming for optimal performance of the detector. 
With the developed threshold trimming procedure the intrinsic threshold dispersion is substantially reduced. Using independent $V_{c m p}$ settings for individual readout chips may potentially improve these results. This would also decrease the minimum achievable threshold since it is limited by the chip with the highest noise level.

Due to the energy dependence of the intrinsic threshold dispersion the current trimming procedure requires trim files at intervals of $2 \mathrm{keV}$ to keep the resulting trimmed dispersions low. A setup consisting of a standard X-ray tube with a monochromator or a fluorescent sample can already provide the necessary monochromaticity but usually lacks the required flux and thus makes trimming very time consuming. Therefore a trimming method producing trim files for any energy from one data set would shorten detector calibration considerably.

To avoid double counting and achieve maximum detection efficiency the pixel threshold needs to be at exactly $50 \%$ of the incident X-ray energy. The threshold can be set above $50 \%$ of the X-ray energy to cut off disturbing fluorescent background at the expense of less efficiency. This can be advantageous for diffuse scattering or phasing experiments. In the case of polychromatic X-rays an energy strip can be cut out of the spectrum by subtracting two images with different threshold. Good threshold dispersion and overall energy resolution are crucial for this kind of experiment. The r. m. s. electronic noise which was measured at room temperature could be further reduced by cooling the module if necessary.

Due to the modularity of the PILATUS system all of the reported methods and results apply for larger module arrays of any size likewise.

\section{ACKNOWLEDGMENT}

The authors would like to thank to M. Naef of DECTRIS, H. Rickert and F. Glaus of PSI for module fabrication. H. Niko from the University of Tokyo, Japan helped to measure the minimum threshold. P. Willmott, R. Herger and D. Martoccia of PSI were instrumental in setting up and collecting data at the SLS Surface Diffraction Station of beamline X04SA. Many thanks to A. Menzel of PSI for helping with the grid experiment at beamline X12SA. We would like to thank R. Horisberger of PSI for advice and valuable discussions.

\section{REFERENCES}

[1] C. Brönnimann, E. F. Eikenberry, B. Henrich, R. Horisberger, G. Huelsen, E. Pohl, B. Schmitt, C. Schulze-Briese, M. Suzuki, T. Tomizaki, H. Toyokawa, and A. Wagner, "The PILATUS $1 \mathrm{M}$ detector," J. Synchrotron Rad., vol. 13, pp. 120-130, 2005.

[2] T. Rohe, C. Brönnimann, F. Glaus, J. Gobrecht, S. Heising, M. Horisberger, R. Horisberger, H. C. Kaestl, J. Lehmann, and S. Streuli, "Developement of an indium bump bond process for silicon pixel detectors at PSI," Nucl. Inst. Meth. Phys. Res. A, vol. 565, pp. 303-308, 2006.

[3] G. Huelsen, C. Brönnimann, E. F. Eikenberry, and A. Wagner, "Protein crystallography with a novel large-area pixel detector," J. Appl. Cryst., vol. 39, pp. 550-557, 2006.

[4] C. Brönnimann, R. Baur, E. F. Eikenberry, S. Kohout, M. Lindner, B. Schmitt, and R. Horisberger, "A pixel read-out chip for the PILATUS project," Nucl. Inst. Meth. Phys. Res. A, vol. 465, pp. 235-239, 2001.

[5] Y. Narumi, K. Kindo, K. Katsumata, M. Kawauchi, C. Brönnimann, U. Staub, H. Toyokawa, Y. Tanaka, A. Kikkawa, T. Yamamoto, M. Hagiwara, T. Ishikawa, and H. Kitamura, "X-ray diffractometer combining synchrotron radiation and pulsed magnetic fields up to 40 T," J. Synchrotron Rad., vol. 13, pp. 271-274, 2006.

[6] P. R. Willmott, S. A. Pauli, R. Herger, C. M. Schlepütz, D. Martoccia, B. D. Patterson, B. Delley, R. Clarke, D. Kumah, C. Cionca, and Y. Yacobi, "Structural basis for the conducting interface between $\mathrm{LaAlO}_{3}$ and $\mathrm{SrTiO}_{3}$," Phys. Rev. Letters, vol. 99, pp. 155502-155502, 2007.

[7] M. Bech, O. Bunk, C. David, P. Kraft, C. Brönnimann, E. F. Eikenberry, and F. Pfeiffer, "X-ray imaging with the PILATUS $100 \mathrm{~K}$ detector," Appl. Radiat. Isot., vol. 66, pp. 474-478, 2008.

[8] F. Pfeiffer, M. Bech, O. Bunk, E. F. Eikenberry, C. Brönnimann, C. Grüzweig, and C. David, "Hard-x-ray dark-field imaging using a grating interferometer," Nature Materials, vol. 7, pp. 134-137, 2008.

[9] G. Anelli, "Conception et Caractérisation de Circuit Intègres Résistants aux Radiations Pour Les Détecteurs de Particules du C en Technologies CMS Submicroniques Profondes," Ph.D. dissertation, Universite Montpellier II, Montpellier, France, 2000.

[10] R. Dinapoli, "A Radiation Tolerant Pixel Detector System for the ALICE and LHCb Experiments at CERN," Ph.D. dissertation, Universite Montpellier II, Montpellier, France, 2004.

[11] X. Llopart, M. Campbell, R. Dinapoli, D. S. Segundo, and E. Pernigotti, "Medipix2: A 64-k pixel readout chip with $55-\mu \mathrm{m}$ square elements working in single photon counting mode," IEEE Trans. Nucl. Sci, vol. 49, pp. 2279-2283, 2002.

[12] P. Kraft, "Characterization of the Readout Chip for the PILATUS $6 \mathrm{M}$ Detector," Diploma, ETH Zürich, Switzerland, 2005.

[13] Hamamatsu Photonics, 2008. [Online]. Available: http://www.hamamatsu.com

[14] R. D. Ryan, "Precision measurements of the ionization energy ant its temperature variation in high purity silicon radiation detectors," IEEE Trans. Nucl. Sci, vol. 20, pp. 473-480, 1973.

[15] $\mathrm{I}^{2} \mathrm{C}$ NXP Semiconductor, 2007, [Online]. Available: http://www.nxp. com/acrobat_download/usermanuals/UM10204_3.pdf

[16] A. Bergamaschi, C. Brönnimann, E. F. Eikenberry, B. Henrich, M. Kobas, P. Kraft, and B. Schmitt, "Experience and results from the 6 megapixel PILATUS system," in Proc. 16th International Workshop on Vertex detectors, Lake Placid, NY, USA, 2007, paper PoS(Vertex 2007)049.

[17] X-123 Spectrometer AMPTEK Inc, 2007 [Online]. Available: http:// www.amptek.com/x123.html

[18] C. Brönnimann, R. Baur, E. F. Eikenberry, P. Fischer, S. Florin, R. Horisberger, M. Lindner, B. Schmitt, and C. Schulze, "A pixel detector for the protein crystallography beamline at the SLS," Nucl. Inst. Meth. Phys. Res. A, vol. 447, pp. 531-535, 2002. 